\title{
BMJ Open Impact of violence against women on severe acute maternal morbidity in the intensive care unit, including neonatal outcomes: a case-control study protocol in a tertiary healthcare facility in Lima, Peru
}

Beatriz Paulina Ayala Quintanilla, ${ }^{1,2,3}$ Wendy E Pollock, ${ }^{1,2,4}$ Susan J McDonald, , ${ }^{1,2}$ Angela J Taft ${ }^{1}$

To cite: Ayala Quintanilla BP Pollock WE, McDonald SJ, et al. Impact of violence against women on severe acute maternal morbidity in the intensive care unit, including neonatal outcomes: a case-control study protocol in a tertiary healthcare facility in Lima, Peru. BMJ Open 2018;8:e020147. doi:10.1136/ bmjopen-2017-020147

- Prepublication history and additional material for this paper are available online. To view these files, please visit the journal online (http://dx.doi. org/10.1136/bmjopen-2017020147).

Received 16 0ctober 2017 Revised 12 February 2018 Accepted 22 February 2018

Check for updates

${ }^{1}$ The Judith Lumley Centre, La Trobe University, Melbourne, Victoria, Australia

${ }^{2}$ Mercy Hospital for Women, Melbourne, Victoria, Australia ${ }^{3}$ Peruvian National Institute of Health, Lima, Peru

${ }^{4}$ University of Melbourne,

Melbourne, Victoria, Australia

Correspondence to

Dr Beatriz Paulina Ayala

Quintanilla;

ayalaquintanilla.b@students.

latrobe.edu.au

\section{ABSTRACT}

Introduction Preventing and reducing violence against women (VAW) and maternal mortality are Sustainable Development Goals. Worldwide, the maternal mortality ratio has fallen about $44 \%$ in the last 25 years, and for one maternal death there are many women affected by severe acute maternal morbidity (SAMM) requiring management in the intensive care unit (ICU). These women represent the most critically ill obstetric patients of the maternal morbidity spectrum and should be studied to complement the review of maternal mortality. VAW has been associated with all-cause maternal deaths, and since many women $(30 \%)$ endure violence usually exerted by their intimate partners and this abuse can be severe during pregnancy, it is important to determine whether it impacts SAMM. Thus, this study aims to investigate the impact of VAW on SAMM in the ICU.

Methods and analysis This will be a prospective casecontrol study undertaken in a tertiary healthcare facility in Lima-Peru, with a sample size of 109 cases (obstetric patients admitted to the ICU) and 109 controls (obstetric patients not admitted to the ICU selected by systematic random sampling). Data on social determinants, medical and obstetric characteristics, VAW, pregnancy and neonatal outcome will be collected through interviews and by extracting information from the medical records using a pretested form. Main outcome will be VAW rate and neonatal mortality rate between cases and controls. VAW will be assessed by using the WHO instrument. Binary logistic followed by stepwise multivariate regression and goodness of fit test will assess any association between VAW and SAMM.

Ethics and dissemination Ethical approval has been granted by the La Trobe University, MelbourneAustralia and the tertiary healthcare facility in LimaPeru. This research follows the WHO ethical and safety recommendations for research on VAW. Findings will be presented at conferences and published in peer-reviewed journals.

\section{Strengths and limitations of this study}

This prospective study will examine for the first time the influence of violence against women on severe acute maternal morbidity (SAMM).

- Studying the stage before maternal death (SAMM) complements the review of maternal mortality.

- This study uses a standardised global instrument for the evaluation of violence against women and a pretested form for the assessment of other variables of interest.

- Studying violence against women can be challenging and may underestimate the rate of abuse due to recall and cultural bias.

- Findings should be interpreted cautiously because this study is within one very large tertiary hospital and further multicentre and multicountry studies may be needed, based on these replicable methods.

\section{INTRODUCTION}

Preventing and reducing violence against women and maternal mortality are sustainable development goals (SDGs). ${ }^{1}$ Worldwide, the maternal mortality ratio (MMR) has declined by $44 \%$ with a reduction from 385 to 216 maternal deaths per 100000 live births between 1990 (532000 maternal deaths) and 2015 (303000 maternal deaths). This equates to approximately 830 women dying daily, of which $99 \%$ of maternal deaths occurred in developing countries. ${ }^{2}$ However, maternal mortality is only a small proportion of the global burden of the maternal morbidity spectrum. ${ }^{3-5}$ This is because for one maternal death there are many women affected by severe acute maternal morbidity (SAMM) during pregnancy, childbirth and the postpartum period ${ }^{5-11}$ including those obstetric 
patients who require multidisciplinary management in the intensive care unit (ICU).$^{11-17}$

ICU admission can be a marker for defining SAMM, ${ }^{1218-25}$ and SAMM can be named as near miss; either term has been widely used to study this population of obstetric patients. ${ }^{1519} 2125-28$ There are no internationally accepted criteria for defining SAMM and its definition may differ across studies, ${ }^{19}{ }^{2025-34}$ and the use of ICU admission to identify severe maternal morbidity has high sensitivity $(86.4 \%)$, specificity $(87.8 \%)$ and positive predicted value (0.85) ${ }^{22} 27{ }^{35-38}$ Women with SAMM treated in the ICU represent the most critically ill obstetric patients ${ }^{11} 1939$ and require timely managed care due to the physiological changes of pregnancy and the care of the mother-baby dyad. ${ }^{11-17} 40-46$

The incidence of maternal ICU admission varies from $0.04 \%$ to $4.54 \%$, and the common causes are mainly direct obstetric clinical conditions. ${ }^{12} 141929$ According to Pollock et $a l,{ }^{14}$ hypertensive disorders were the leading cause of ICU admission ( $0.09 \%$ of deliveries), followed by obstetric haemorrhage $(0.07 \%)$ and sepsis $(0.02 \%)$, and although the ICU admission profile was similar worldwide there were higher rates of maternal deaths in the ICUs of developing countries.

Violence against women is a global public health problem and many women of reproductive age endure violence usually exerted by their intimate partners (intimate partner violence (IPV)). Globally, 30\% of women have experienced partner abuse. ${ }^{47-49}$ However, the IPV rate varies across studies ranging from $15 \%$ to $71 \%$ and from $1 \%$ to $28 \%$ during pregnancy. ${ }^{50}$ Diverse studies have also reported a wide range of IPV rate during pregnancy from $0.9 \%$ to $20.1 \%$ in high-income countries ${ }^{51} 3 \%$ to $44 \%$ in Latin America and Caribbean countries ${ }^{52}$ and 2\% to $57 \%$ in African countries. ${ }^{53}$

Several studies have reported negative and mortal consequences of IPV on reproductive age women. ${ }^{54-57}$ During pregnancy, these adverse health outcomes affect the mother-baby dyad and can be augmented by consequent risky health behaviours (smoking, alcohol consumption, substance abuse, poor nutrition, lack of seeking healthcare, among others $)^{57-60}$ and physiological mechanisms through neural, neuroendocrine and immune responses to acute and/or chronic stress originating from exposure to violence. ${ }^{5561}$ All of this may exacerbate pre-existing medical conditions and/or lead to diverse pregnancy complications. ${ }^{52} 556162$ Even though women could be more vulnerable to IPV during the pregnancy and puerperium periods, no data on violence against women of obstetric patients in the ICU have been reported in Peru or elsewhere. ${ }^{63}$ Thus, it is important to examine whether violence against women is a risk factor for women with SAMM. We are conducting this study in Peru, an upper middle-income country ${ }^{64}$ with a lifetime IPV rate of $68.2 \%$ (emotional $64.2 \%$, physical $31.7 \%$ and sexual $6.6 \%) .{ }^{65}$ In Peru, there are about 9 cases of femicide monthly ${ }^{56}$ and an MMR of 68, which fell $72.9 \%$ from 251 between 1990 and $2015 .^{2}$
There has been an increased concern about the negative influence of violence against women on maternal mortality, ${ }^{66-72}$ and violence against women has been associated with all-cause maternal deaths. ${ }^{66} 67$ Although, this association was first analysed 16 years ago, ${ }^{67}$ there is still a paucity of studies investigating the influence of violence against women on SAMM, ${ }^{63}$ which can be considered as a complement to a review of maternal deaths. ${ }^{1522} 2936$ 73-75 Thus, it is important to determine whether violence against women impacts SAMM, since many women endure violence usually exerted by their intimate partners and this abuse can be severe during pregnancy. Therefore, this research of the stage before maternal death (SAMM) will provide for the first time, a better understanding about what potential factors, such as violence against women, are affecting obstetric patients with SAMM in the ICU. This will make an important contribution to global knowledge of causes of maternal morbidity.

\section{HYPOTHESIS}

We hypothesise that violence against women is significantly associated with severe acute maternal morbidity in obstetric patients managed in the ICU.

\section{OBJECTIVES}

- To investigate the impact of violence against women on obstetric patients with SAMM treated in the ICU (cases) by comparing them with obstetric patients not admitted to the ICU (controls) in a tertiary healthcare facility in Lima,Peru;

- To evaluate pregnancy and neonatal outcome of women with SAMM in the ICU of a tertiary healthcare facility in Lima, Peru.

\section{METHODS}

This is a prospective case-control study protocol and follows the Strengthening the Reporting of Observational Studies in Epidemiology (STROBE) criteria. ${ }^{76} 77$

\section{Study design}

In this case-control study, cases will be obstetric patients experiencing SAMM in the ICU(s) including miscarriage, therapeutic abortion, unsafe abortion and ectopic pregnancy prospectively identified from the ICU register. The inclusion criteria of cases include: (1) a woman with a maternal ICU admission due to a complication(s) during pregnancy, delivery or within 42 days of the ending of pregnancy, with favourable evolution and who fulfils eligibility prior to hospital discharge; (2) 18-year-old or older; (3) Spanish speaker and (4) gives written informed consent. The exclusion criteria are: (1) a woman with mental illness or disabilities or other similar disabling pathologies; (2) not able to provide informed consent; (3) an obstetric patient referred from other healthcare facilities for maternity care and (4) ICU stay less than 24 hours. The controls are from the same source population that 
gives rise to the cases and their inclusion criteria consist of (1) a woman admitted to this hospital during pregnancy, delivery or within 42 days of the ending of pregnancy, with favourable evolution and who fulfils eligibility prior to hospital discharge; (2) 18-year-old or older; (3) Spanish speaker and (iv) gives written informed consent. The exclusion criteria are: (1) a woman with mental illness or disabilities or other similar disabling pathologies; (2) not able to provide informed consent and (3) an obstetric patient referred from other healthcare facilities for maternity care. Favourable evolution means that an obstetric patient has received the required management for the condition responsible for her admission to the hospital or the ICU, is haemodynamically stable without requiring strict monitoring or specialised treatment, her life is not at risk and is well enough to be discharge from the hospital to home (it implies that the maternal morbidity has resolved for the cases).

We plan to include all cases who meet the selection criteria during the study period, until we reach the estimated sample size of 109 obstetric patients admitted to the ICU. This is because there were 263 ICU admissions (pregnant and non-pregnant women) in 2012, 230 in 2013 and 274 in 2014 according to the Department of Epidemiology of this hospital ${ }^{78}$ and, approximately $48 \%$ of the ICU admissions are referred from other healthcare facilities (as indicated by the chief of the ICU). We expect that cases will be recruited during a period of 12 months, though recruitment will continue for longer if necessary for the minimum sample sizes to be achieved. Controls will be selected by using a probability sampling method, specifically a systematic random sampling (without replacement) starting with 18 and with a value of $\mathrm{k}=131$ as the sampling interval.

\section{Setting}

This study is being undertaken in a tertiary hospital located in the capital city of Peru (where about one-third of the Peruvian population live). This institution is the main national referral hospital for high-risk maternal and neonatal patients throughout Peru, where there are over 22000 deliveries yearly, which equates to approximately 1 delivery every $25 \mathrm{~min}^{79}$

\section{Sample size}

The sample size will comprise 218 participants (109 controls and 109 cases) which has been calculated by using the Sample Size Calculation for Unmatched CaseControl Studies of the software open $\mathrm{Epi}^{80}$ with a $95 \%$ CI and a power of $80 \%$ to detect a $(18.8 \%)$ difference in the exposure of violence against women (rate) between cases and controls. There are not preceding studies assessing the prevalence of violence against women among obstetric patients with SAMM in the ICU. We estimated an IPV rate of $24.3 \%$ for controls and $43.1 \%$ for cases in accordance with previous research investigating the relationship between IPV and preeclampsia in Peruvian pregnant women at this maternity hospital. ${ }^{81}$

\section{Recruitment method}

Participants will be invited to participate and recruited during their hospital stay once their acute medical condition(s) have been resolved (this will be after they were discharged from the ICU for the case women) and before they are discharged from hospital to going home. Women in the control group will be invited to participate within 1 week of a case woman being interviewed. The researcher (interviewer), a Spanish-speaking midwife, has been trained and has research experience working on violence against women studies and research ethics. The researcher will check with the hospital staff regarding eligibility (selection criteria, eg, mental illness and others), prior to approaching and inviting potential participants to the study. She will also ensure that the potential participants understand the participant information statement and answer all their questions and explain to the participants that they can refuse or withdraw their participation at any time without affecting their healthcare and rights.

\section{Data collection}

Data collection commenced on 23 October 2015 and is still ongoing. We expect to finish by the end of March 2018. Currently, we are collecting data from the medical records. Women who give consent will be interviewed once in private using a structured questionnaire (see online supplementary appendix 1 ). We will also extract information from their medical records using a pretested form developed by the team members (see online supplementary appendix 2).

\section{Interview}

The following information will be obtained during the face-to-face interview: sociodemographic characteristics (age, place of residence, educational level, marital status, occupation and type of health insurance) of the participant and her partner; behavioural factors (smoking, use of alcohol or drugs); medical (previous and current diseases) and obstetric characteristics (number of pregnancies, previous abortions, prenatal care visits, previous caesarean sections and vaginal births; use of family planning methods; anaemia during pregnancy and use of iron supplements during pregnancy) and violence against women evaluation.

Violence against women will be assessed by using questions from a standardised instrument of the WHO. ${ }^{50}$ These questions were validated, translated and adapted rigorously, since Peru was one of the countries selected in the WHO multicountry study instrument. ${ }^{50}$ They have been also used by other researchers for investigating violence against women in Peru, and an adapted version was applied in the Peruvian Demographic and Family Health Survey. ${ }^{65}$

Violence against women evaluation will comprise emotional (including controlling behaviours), physical and sexual abuse exerted by intimate partners and by non-intimate partners (relatives, friends or others) assessed 12 months before and during pregnancy. The 
participants will answer the frequency (once or twice or many) for each act of emotional, physical or sexual violence. Violence against women will be examined in an empathetic, supportive and non-judgemental manner, giving the participants the opportunity not to answer any questions that make them feel uncomfortable or to reschedule or terminate the interview at any time. The interview will only be conducted with the woman alone and in private. If the interview is interrupted, the interviewer will change the topic or may terminate the interview correspondingly. It is expected that the interview will take approximately $25-30 \mathrm{~min}$. After the interview, information about free social support services for domestic violence (available at this maternity hospital) will be offered to every participant including a referral if they wish. There will be also debriefing sessions for the well-being of the interviewer. Individual support/counselling can be arranged at the hospital for the interviewer or participant if it is needed.

\section{Extracting data from medical records}

We will extract data from the medical reports of the mothers and newborns (if applicable) related to SAMM, pregnancy outcome, fetal and neonatal characteristics and outcome.

SAMM data will include hospital admission date, clinical causes for hospitalisation and/or ICU admission, diagnoses after being discharged from the ICU and/or the hospital, number of hospital stay days and ICU stay days, type of delivery, additional procedures, weeks of pregnancy when SAMM occurs, organ failure(s), use of technologies (mechanical ventilation, transfusion, use of inotropic support and renal replacement therapies), main delays (in relation to the 3 delays model framework used to study maternal mortality ${ }^{82}$ ) and pregnancy outcome (see online supplementary appendix 2).

Fetal and neonatal data will include: birth weight; birth age; sex; Apgar score (at 1 and $5 \mathrm{~min}$ ); outcome at birth; clinical cause(s) for neonatal ICU (NICU); number of days at the NICU (if applicable) or at the hospital; use of technologies and/or antibiotics at the NICU; feeding type (breastfeeding, formula or both); discharge status and diagnoses from the hospital and/or the NICU (see online supplementary appendix 2 ).

\section{Data entry and analysis}

Data will be entered using SPSS V.24.0. Univariate analysis will be carried out to describe the characteristics of SAMM, social determinants, violence against women and pregnancy and neonatal outcomes. Quantitative variables will be displayed as the mean $\pm \mathrm{SD}$ and/or median (interquartile rate) after verifying their normal or asymmetrical distribution. Difference of means analyses between variables will be performed using appropriate statistical tests (Student's t-test or Mann-Whitney U test or other similar tests). Qualitative variables will be displayed as frequencies.
We will apply a bivariate analysis to evaluate the association of violence against women (partner and non-partner) as an independent variable with SAMM; and we will also evaluate this association with partner violence only and with non-partner violence only.

We will fully assess violence against women as a risk factor by using a multivariate logistic regression modelling considering an OR with 95\% CI. Crude ORs will also be calculated. Statistical significance will be set at $\mathrm{p}<0.05$ for all analyses. We will identify effect modification for age, level of education, alcohol consumption and use of drugs by performing a stratified analysis of these variables that could potentially modify the effect of violence against women on SAMM. This will be assessed by the calculation of crude ORs within every level of each variable that is stratified. The final model will include the sociodemographic characteristics of the participants, other variables of interest (such as household income, partner's educational level, partner's occupation, etc) according to the literature review and modifiers (we will remove the least significant modifiers one at a time until only those with $p<0.05$ remain). Those variables with $p$ values of $<0.25$ will be initially chosen to be included in the model ${ }^{83}$ and the Hosmer-Lemeshow's goodness-of-fit test will be used to assess model adequacy and stepwise multiple regression analysis performed to select and identify the predictive factors in the final model. ${ }^{83}$

\section{ETHICS AND DISSEMINATION}

Ethical approval has been granted by the La Trobe University Human Ethics committee (HEC15-023), Melbourne, Australia and the Institutional Review Board of the tertiary healthcare hospital in Lima, Peru. Individual written informed consent will be obtained from participants prior to data collection. The present research follows the WHO and other ethical and safety recommendations for research on gender-based violence to ensure the safety of the participants and the interviewer. ${ }^{50} 84-88$

It is planned that the findings of this case-control study will be presented at La Trobe University and national and/or international conferences, and it will be also published in a peer-reviewed journal. It is expected that these findings will inform policy-makers, patients and the public through these presentations.

\section{PRESENTING AND REPORTING RESULTS}

This prospective case-control study will examine for the first time the influence of violence against women on obstetric patients affected by severe acute maternal morbidity who require management in the ICU and evaluate their pregnancy and neonatal outcomes. We will present major findings in tables and also describe results in narrative format outlining effect sizes and their parameters. The findings and further publications will be reported following the STROBE criteria, ${ }^{76}$ which is 
part of the Enhancing the QUAlity and Transparency Of health Research Network website.

\section{DISCUSSION}

The influence of violence against pregnant women on the incidence of or type of SAMM is not known and worthy of study. Although, the negative contribution of violence against women to maternal deaths was described in the 1997-1999 Confidential Enquiry into Maternal Death, ${ }^{67}$ and many studies have reported negative and fatal repercussions of IPV on women's health including during all stages of pregnancy, ${ }^{52-62}$ there is a paucity of studies assessing the relationship between violence against women and SAMM. ${ }^{63}$ Investigating women affected by SAMM could complement the review of maternal deaths ${ }^{1522} 293673-75$ to understand which underlying factors are influencing the sequence of events from a healthy pregnancy through minor complications to life-threatening obstetric conditions and even death in childbearing women. $^{26} 8990$

This case-control study will contribute to evidence about the potential negative consequences of violence against women of obstetric patients with SAMM in the ICU, who represent the most critically ill patients ${ }^{11} 1939$ and have been shown to be an important component of the maternal morbidity spectrum requiring timely managed care. ${ }^{11-17} 40-46$ This may help to highlight that non-biological factors (violence against women), which are potentially modifiable, may be associated with SAMM and are important to address to reduce maternal morbidity-mortality in Peru and in other low/middle-income countries. Acute or chronic exposure to violence adversely affects both the mothers and their babies and can be augmented by any risky health behaviours ${ }^{57-60}$ and through physiological (neural, neuroendocrine and immune) mechanisms in response to this abuse. ${ }^{5561}$ All of this may exacerbate pre-existing medical conditions and/ or lead to diverse pregnancy complications. ${ }^{52556162}$ Thus, it would be important to know if violence against women is a risk factor for SAMM in the ICU and to understand the complete picture of the global burden of maternal morbidity-mortality to improve mother-baby dyad health and women's well-being.

This study may also help participants affected by violence to disclose abuse in a safe and supportive environment and provide an opportunity for those abused women to understand that it is possible to prevent violence and improve their and other women's lives, since they will be informed and/or referred to the free social support services available for domestic violence provided by the Peruvian government.

We understand that the findings of this study should be interpreted cautiously and some limitations should be noted. First, this research will be undertaken in a single centre. It will be important to carry out further multicentre and multicountry studies. Other limitations may include recall, cultural and measurement bias which can underestimated or overestimate the exposure. ${ }^{5091}$ This is because the assessment of violence against women is complex and challenging. Accordingly, this research follows the ethical and safety recommendations for research on violence against women ${ }^{50-88}$ and uses standardised questions for the assessment of violence against women (WHO instrument) and a pretested questionnaire for the evaluation of other factors. Amendments to this protocol are not expected. However, if any are required, these amendments will be reported transparently.

Notwithstanding these limitations, we hope that this study will contribute to the global effort towards achieving SDGs by providing valuable information for a better understanding of SAMM and violence against women in Peru. This will make an important contribution to global knowledge of causes of maternal morbidity by providing evidence of the relationship between violence against women and SAMM, which is important for preventing and/or reducing maternal morbidity-mortality and improve maternal health. Therefore, we expect that this research will extend knowledge in an identified research gap and may provide direction for further studies in obstetric women affected by SAMM in the ICU.

Contributors BPAQ, AT, SJM and WP conceived and designed the study protocol. This study is part of the first author PhD thesis. BPAQ drafted the manuscript and all authors edited following versions of the draft. BPAQ, AT, SJM and WP revised critically the methodological and clinical content of the protocol to make contributions. All authors reviewed and approved the final manuscript.

Funding This study is part of a doctoral study at La Trobe University was supported by the Peruvian Government through PRONABEC (National Program of Scholarship and Educational Loan) and La Trobe University. No funding bodies will have any role in study design, collecting data, analysing data, interpreting findings and writing, reviewing or deciding to publish the manuscript.

Competing interests None declared.

Patient consent Detail has been removed from this case description/these case descriptions to ensure anonymity. The editors and reviewers have seen the detailed information available and are satisfied that the information backs up the case the authors are making.

Ethics approval Ethical approval has been granted by the La Trobe University Human Ethics Committee, Melbourne, Australia (HEC15-023) and the Institutional Review Board of tertiary healthcare facility in Lima, Peru.

Provenance and peer review Not commissioned; externally peer reviewed.

Open Access This is an Open Access article distributed in accordance with the Creative Commons Attribution Non Commercial (CC BY-NC 4.0) license, which permits others to distribute, remix, adapt, build upon this work non-commercially, and license their derivative works on different terms, provided the original work is properly cited and the use is non-commercial. See: http://creativecommons.org/ licenses/by-nc/4.0/

(c) Article author(s) (or their employer(s) unless otherwise stated in the text of the article) 2018. All rights reserved. No commercial use is permitted unless otherwise expressly granted.

\section{REFERENCES}

1. United Nations. Transforming our world: the 2030 Agenda for Sustainable Development. 2015. Resolution adopted by the General Assembly on 25 September 2015. https://sustainabledevelopment. un.org/post2015/transformingourworld (accessed 6 May 2017).

2. World Health Organization. Trends in maternal mortality: 1990 to 2015. Estimates by WHO, UNICEF, UNFPA, World Bank Group and the United Nations Population Division. 2015 http://www.who.int/ classifications/icd/factsheet/en/ (accessed 15 Feb 2016). 
3. Mantel GD, Buchmann E, Rees $\mathrm{H}$, et al. Severe acute maternal morbidity: a pilot study of a definition for a near-miss. $\mathrm{Br} \mathrm{J}$ Obstet Gynaecol 1998;105:985-90.

4. Geller SE, Cox SM, Callaghan WM, et al. Morbidity and mortality in pregnancy: laying the groundwork for safe motherhood. Womens Health Issues 2006;16:176-88.

5. Firoz T, Chou D, von Dadelszen $P$, et al. Measuring maternal health: focus on maternal morbidity. Bull World Health Organ 2013;91:794-6.

6. Ashford L. Hidden suffering: disabilities from pregnancy and childbirth in less developed countries. 2002 http://www.prb.org/pdf/ hiddensufferingeng.pdf (accessed 23 Jul 2014).

7. Fottrell E, Kanhonou L, Goufodji S, et al. Risk of psychological distress following severe obstetric complications in Benin: the role of economics, physical health and spousal abuse. Br J Psychiatry 2010;196:18-25.

8. Pacagnella RC, Cecatti JG, Camargo RP, et al. Rationale for a longterm evaluation of the consequences of potentially life-threatening maternal conditions and maternal "near-miss" incidents using a multidimensional approach. J Obstet Gynaecol Can 2010;32:730-8.

9. Wilson RE, Salihu HM. The paradox of obstetric "near misses": converting maternal mortality into morbidity. Int J Fertil Womens Med 2007;52:121-7.

10. Storeng KT, Drabo S, Ganaba R, et al. Mortality after near-miss obstetric complications in Burkina Faso: medical, social and healthcare factors. Bull World Health Organ 2012;90:418-25.

11. Plaat F, Naik M. Critical care in pregnancy. Crit Care 2011;15:1014.

12. Zieleskiewicz L, Chantry A, Duclos G, et al. Intensive care and pregnancy: epidemiology and general principles of management of obstetrics ICU patients during pregnancy. Anaesth Crit Care Pain Med 2016;35(Suppl 1):S51-7.

13. Guntupalli KK, Hall N, Karnad DR, et al. Critical illness in pregnancy: part I: an approach to a pregnant patient in the ICU and common obstetric disorders. Chest 2015;148:1093-104.

14. Pollock W, Rose L, Dennis CL. Pregnant and postpartum admissions to the intensive care unit: a systematic review. Intensive Care Med 2010;36:1465-74.

15. Baskett TF. Epidemiology of obstetric critical care. Best Pract Res Clin Obstet Gynaecol 2008;22:763-74.

16. Martin SR, Foley MR. Intensive care in obstetrics: an evidence-based review. Am J Obstet Gynecol 2006;195:673-89.

17. Germain S, Wyncoll D, Nelson-Piercy C. Management of the critically ill obstetric patient. Curr Obstet Gynaecol 2006;16:125-33.

18. Oud L. Epidemiology of pregnancy-associated ICU utilization in Texas: 2001-2010. J Clin Med Res 2017:9:143-53.

19. Senanayake H, Dias T, Jayawardena A. Maternal mortality and morbidity: epidemiology of intensive care admissions in pregnancy. Best Pract Res Clin Obstet Gynaecol 2013;27:811-20.

20. Bouvier-Colle MH, Mohangoo AD, Gissler M, et al. What about the mothers? An analysis of maternal mortality and morbidity in perinatal health surveillance systems in Europe. BJOG 2012;119:880-90.

21. Reichenheim ME, Zylbersztajn F, Moraes CL, et al. Severe acute obstetric morbidity (near-miss): a review of the relative use of its diagnostic indicators. Arch Gynecol Obstet 2009;280:337-43.

22. Hirshberg A, Srinivas SK. Epidemiology of maternal morbidity and mortality. Semin Perinatol 2017;41:332-7.

23. Brace V, Penney G, Hall M. Quantifying severe maternal morbidity: a Scottish population study. BJOG 2004;111:481-4.

24. Lawton BA, Wilson LF, Dinsdale RA, et al. Audit of severe acute maternal morbidity describing reasons for transfer and potential preventability of admissions to ICU. Aust N Z J Obstet Gynaecol 2010;50:346-51.

25. Chantry AA, Deneux-Tharaux C, Bonnet MP, et al. Pregnancy-related ICU admissions in France: trends in rate and severity, 2006-2009. Crit Care Med 2015;43:78-86.

26. Say L, Souza JP, Pattinson RC; WHO Working Group on Maternal Mortality and Morbidity Classifications. Maternal near miss--towards a standard tool for monitoring quality of maternal health care. Best Pract Res Clin Obstet Gynaecol 2009;23:287-96.

27. Callaghan WM, Grobman WA, Kilpatrick SJ, et al. Facility-based identification of women with severe maternal morbidity: it is time to start. Obstet Gynecol 2014;123:978-81.

28. Donati S, Senatore S, Ronconi A, et al. Obstetric near-miss cases among women admitted to intensive care units in Italy. Acta Obstet Gynecol Scand 2012;91:452-7.

29. Tunçalp O, Hindin MJ, Souza JP, et al. The prevalence of maternal near miss: a systematic review. BJOG 2012;119:653-61.

30. Lewis G. Reviewing maternal deaths to make pregnancy safer. Best Pract Res Clin Obstet Gynaecol 2008;22:447-63.

31. Souza JP, Cecatti JG, Parpinelli MA, et al. Appropriate criteria for identification of near-miss maternal morbidity in tertiary care facilities: a cross sectional study. BMC Pregnancy Childbirth 2007;7:20.
32. van Roosmalen J, Zwart J. Severe acute maternal morbidity in high-income countries. Best Pract Res Clin Obstet Gynaecol 2009;23:297-304.

33. David E, Machungo F, Zanconato G, et al. Maternal near miss and maternal deaths in Mozambique: a cross-sectional, region-wide study of 635 consecutive cases assisted in health facilities of Maputo province. BMC Pregnancy Childbirth 2014;14:401.

34. You WB, Chandrasekaran S, Sullivan J, et al. Validation of a scoring system to identify women with near-miss maternal morbidity. Am J Perinatol 2013;30:21-4.

35. Kilpatrick SJ, Berg C, Bernstein P, et al. Standardized severe maternal morbidity review: rationale and process. Obstet Gynecol 2014;124:361-6.

36. Kilpatrick SK, Ecker JL; American College of Obstetricians and Gynecologists and the Society for Maternal-Fetal Medicine. Severe maternal morbidity: screening and review. Am J Obstet Gynecol 2016;215:B17-22.

37. Geller SE, Rosenberg D, Cox S, et al. A scoring system identified near-miss maternal morbidity during pregnancy. J Clin Epidemiol 2004:57:716-20.

38. Main EK, Abreo A, McNulty J, et al. Measuring severe maternal morbidity: validation of potential measures. Am J Obstet Gynecol 2016;214:643.e1-643.e10.

39. Zwart JJ, Dupuis JR, Richters A, et al. Obstetric intensive care unit admission: a 2-year nationwide population-based cohort study. Intensive Care Med 2010;36:256-63.

40. Shapiro JM. Critical care of the obstetric patient. J Intensive Care Med 2006;21:278-86.

41. Honiden S, Abdel-Razeq SS, Siegel MD. The management of the critically ill obstetric patient. J Intensive Care Med 2013;28:93-106.

42. Neligan PJ, Laffey JG. Clinical review: special populations-critical illness and pregnancy. Crit Care 2011;15:227.

43. Soubra SH, Guntupalli KK. Critical illness in pregnancy: an overview. Crit Care Med 2005;33(10 Suppl):S248-55.

44. Trikha A, Singh P. The critically ill obstetric patient-recent concepts. Indian J Anaesth 2010;54:421-7.

45. Aoyama K, Seaward PG, Lapinsky SE. Fetal outcome in the critically ill pregnant woman. Crit Care 2014;18:307.

46. Munnur U, Bandi V, Guntupalli KK. Management principles of the critically ill obstetric patient. Clin Chest Med 2011;32:53-60.

47. World Health Organization. Global and regional estimates of violence against women: prevalence and health effects of intimate partner violence and non-partner sexual violence. $2013 \mathrm{http}: / /$ apps.who. int/iris/bitstream/10665/85239/1/9789241564625_eng.pdf?ua=1 (accessed 13 Nov 2014).

48. Devries KM, Mak JY, García-Moreno C, et al. Global health. The global prevalence of intimate partner violence against women. Science 2013;340:1527-8

49. World Health Organization. The Global status report on violence prevention 2014. 2014 http://www.who.int/violence_injury prevention/violence/status_report/2014/en/ (accessed 28 May 2015).

50. Garcia-Moreno C, Jansen HA, Ellsberg M, et al. Prevalence of intimate partner violence: findings from the WHO multi-country study on women's health and domestic violence. Lancet 2006;368:1260-9.

51. Gazmararian JA, Lazorick S, Spitz AM, et al. Prevalence of violence against pregnant women. JAMA 1996;275:1915-20.

52. Han A, Stewart DE. Maternal and fetal outcomes of intimate partner violence associated with pregnancy in the Latin American and Caribbean region. Int J Gynaecol Obstet 2014;124:6-11.

53. Shamu S, Abrahams N, Temmerman M, et al. A systematic review of African studies on intimate partner violence against pregnant women: prevalence and risk factors. PLoS One 2011;6:e17591.

54. Stöckl H, Devries K, Rotstein A, et al. The global prevalence of intimate partner homicide: a systematic review. Lancet 2013;382:859-65.

55. Black MC. Intimate partner violence and adverse health consequences: implications for clinicians. J Womens Health 2011;5:428-39.

56. Ayala Quintanilla BP, Taft A, McDonald S, et al. An examination of femicides in Peru between 2009 and 2014. Int J Gynaecol Obstet 2016;134:342-3.

57. Sugg N. Intimate partner violence: prevalence, health consequences, and intervention. Med Clin North Am 2015;99:629-49.

58. Sarkar NN. The impact of intimate partner violence on women's reproductive health and pregnancy outcome. J Obstet Gynaecol 2008:28:266-71.

59. Chambliss LR. Intimate partner violence and its implication for pregnancy. Clin Obstet Gynecol 2008;51:385-97.

60. Chisholm CA, Bullock L, Ferguson JEJ. Intimate partner violence and pregnancy: epidemiology and impact. Am J Obstet Gynecol 2017;217:141-4. 
61. Alhusen JL, Ray E, Sharps $P$, et al. Intimate partner violence during pregnancy: maternal and neonatal outcomes. $J$ Womens Health 2015;24:100-6.

62. Hill A, Pallitto C, McCleary-Sills J, et al. A systematic review and meta-analysis of intimate partner violence during pregnancy and selected birth outcomes. Int J Gynaecol Obstet 2016;133:269-76.

63. Ayala Quintanilla BP, Taft A, McDonald S, et al. Social determinants and maternal exposure to intimate partner violence of obstetric patients with severe maternal morbidity in the intensive care unit: a systematic review protocol. BMJ Open 2016;6:e013270.

64. The World Bank. Data. 2017 http://data.worldbank.org/about/ country-and-lending-groups (accessed 4 Jun 2017).

65. Instituto Nacional De Estadistica E Informatica. Encuesta Demografica y de Salud Familiar 2016 - Nacional y Regional 2016. 2017 https://www.inei.gob.pe/media/MenuRecursivo/publicaciones_ digitales/Est/Lib1356/

66. Lewis G. The Confidential Enquiry into Maternal and Child Health (CEMACH). Saving Mothers' Lives: reviewing maternal deaths to make motherhood safer - 2003-2005. The seventh report on confidential enquiries into maternal deaths in the United Kingdom. London: CEMACH, 2007. http://www.publichealth.hscni.net/sites/ default/files/Saving\%20Mothers\%27\%20Lives\%202003-05\%20.pdf. (accessed 12 Dec 2017).

67. The Confidential Enquiries into Maternal Deaths in the United Kingdom. Why mothers die 1997-1999. The fifth report of the confidential enquiries into maternal deaths. $2001 \mathrm{http}: / / \mathrm{www}$. hqip.org.uk/national-programmes/a-z-of-clinical-outcome-reviewprogrammes/cmace-reports/ (accessed 2 Aug 2014).

68. Knight M, Tuffnell DJ, Kenyon S, et al; Saving lives, improving mothers' care - Surveillance of maternal deaths in the UK 2011-13 and lessons learned to inform maternity care from the UK and Ireland confidential enquiries into maternal deaths and morbidity 2009-13. Oxford: National Perinatal Epidemiology Unit, University of Oxford, 2015. https://www.npeu.ox.ac.uk/ (accessed 15 Apr 2016).

69. Knight M, Kenyon S, Brocklehurst P, et al; Saving lives, improving mothers' care - Lessons learned to inform future maternity care from the UK and Ireland confidential enquiries into maternal deaths and morbidity 2009 -12. Oxford: National Perinatal Epidemiology Unit, University of Oxford, 2014. https://www.npeu.ox.ac.uk/downloads/ files/mbrrace-uk/reports/Saving\%20Lives\%20lmproving\% 20Mothers\%20Care\%20report\%202014\%20Full.pdf (accessed 27 Apr 2016).

70. United Nation, Every Woman Every Child. EVERY NEWBORN. An Action Plan To End Preventable Deaths. 2014 http://apps.who. int/iris/bitstream/10665/127938/1/9789241507448 eng.pdf?ua=1 (accessed 14 Mar 2016).

71. Farquhar C, Sadler L, Masson V, et al. Beyond the numbers: classifying contributory factors and potentially avoidable maternal deaths in New Zealand, 2006-2009. Am J Obstet Gynecol 2011:205:331.e1-331.e8.

72. Murphy NJ, Quinlan JD. Trauma in pregnancy: assessment, management, and prevention. Am Fam Physician 2014;90:717-22.

73. Knight $\mathrm{M}$, Lewis $\mathrm{G}$, Acosta $\mathrm{CD}$, et al. Maternal near-miss case reviews: the UK approach. BJOG 2014;121(Suppl 4):112-6.

74. Ananth CV, Smulian JC. Chapter 1 epidemiology of critical illness in pregnancy. In: Belfort MA, Saade G, Foley MR, et al. Critical care obstetrics. Fifth Edition, 2011.

75. Knight $\mathrm{M}$, Acosta $\mathrm{C}$, Brocklehurst $\mathrm{P}$, et al. Beyond maternal death: improving the quality of maternal care through national studies of 'near-miss' maternal morbidity. Programme Grants for Applied Research 2016;4:1-180.

76. von Elm E, Altman DG, Egger M, et al. The Strengthening the Reporting of Observational Studies in Epidemiology (STROBE) statement: guidelines for reporting observational studies. The Lancet 2007;370:1453-7.

77. von Elm E, Altman DG, Egger M, et al. The Strengthening the Reporting of Observational Studies in Epidemiology (STROBE) Statement: guidelines for reporting observational studies. Int J Surg 2014:12:1495-9.

78. Instituto Nacional Materno Perinatal. Boletín Estadístico 2014. 2014 http://www.inmp.gob.pe/institucional//sala-situacional/1421334856 (accessed 28 Jan 2015)

79. Instituto Nacional Materno Perinatal. Boletín Estadístico 2015 - 2016 2016 http://www.inmp.gob.pe/institucional/boletines-estadisticos/ 1422371837 (accessed 28 May 2017).

80. Open Epi. Sample Size Calculation for Unmatched Case-Control Studies. 2014. Version 3.03.17 http://web1.sph.emory.edu/cdckms/ sample\%20size\%202\%20grps\%20case\%20control.html (accessed 2 Jul 2015).

81. Sanchez SE, Qiu C, Perales MT, et al. Intimate partner violence (IPV) and preeclampsia among Peruvian women. Eur J Obstet Gynecol Reprod Biol 2008;137:50-5.

82. Thaddeus S, Maine D. Too far to walk: maternal mortality in context. Soc Sci Med 1994;38:1091-110.

83. Hosmer DV, Lemeshow S. Applied logistic regression. Second Edition. USA: A Wiley-Interscience Publication, 2000:375.

84. Jewkes R, Dartnall E, Sikweyiya Y. Ethical and safety recommendations for research on the perpetration of sexual violence. Sexual Violence Research Initiative Pretoria, South Africa: Medical Research Council, 2012. http://www.svri.org/EthicalRecommendati ons.pdf (accessed 28 May 2015).

85. Ellsberg M, Heise L. Researching Violence Against Women: A Practical Guide for Researchers and Activists. Washington DC, United States: World Health Organizationb PATH, 2005. http:// whqlibdoc.who.int/publications/2005/9241546476_eng.pdf?ua=1 (accessed 26 May 2015).

86. World Health Organization. Putting Women First: Ethical and Safety Recommendations for Research on Domestic Violence Against Women. 2001 http://www.who.int/gender/violence/womenfirtseng. pdf (accessed 27 May 2015).

87. Jansen $\mathrm{H}$, Watts $\mathrm{C}$, Ellsberg $\mathrm{M}$, et al. Interviewer Training in the WHO Multi-Country Study on Women's Health and Domestic Violence. $2004 \mathrm{http} / / /$ www.who.int/gender/documents/Interviewer_training.pdf (accessed 27 May 2015).

88. Garcia-Moreno C, Jansen H, Ellsberg M, et al. WHO Multicountry Study on Women's Health and Domestic Violence Against Women. Initial results on prevalence, health outcomes and women's responses. 2005 http://www.who.int/reproductivehealth/publications/ violence/24159358X/en/ (accessed 2 Jul 2014).

89. Karolinski A, Mercer R, Micone P, et al. The epidemiology of lifethreatening complications associated with reproductive process in public hospitals in Argentina. BJOG 2013;120:1685-95.

90. Filippi V, Chou D, Ronsmans C, et al; Levels and causes of maternal mortality and morbidity. In: Black RE, Laxminarayan R, Temmerman M, Reproductive, maternal, newborn, and child health. Disease control priorities. third edition. Washington, DC: World Bank, 2016: 2.

91. Pandis N. Case-control studies: part 2. Am J Orthod Dentofacial Orthop 2014;146:402-3. 